\title{
Levels of Interleukin-17 and Interleukin-23 in Patients with Systemic Lupus Erythematosus (SLE) in Trinidad and Tobago
}

\author{
Alisa Nobee ${ }^{1}$, Angel Justiz-Vaillant ${ }^{1}{ }^{*}$, Patrick E Akpaka' ${ }^{1}$ and Peter Poon-King ${ }^{2}$ \\ ${ }^{1}$ Department of Para-Clinical Sciences, Faculty of Medical Sciences, The University of the West Indies, Trinidad and Tobago, West Indies \\ ${ }^{2}$ San Fernando General Hospital, San Fernando, Trinidad and Tobago, West Indies
}

\begin{abstract}
Serum interleukin (IL)-17 and IL-23 and their association with systemic lupus erythematosus (SLE) is not well understood. A prospective cross sectional and observational study was done using twenty eight (28) SLE patients and fourteen (14) non SLE control subjects. Blood samples were taken to delineate the presence of IL-17 and IL-23 using ELISA among patients of the Rheumatology out-patient clinic at the San Fernando General Hospital, Trinidad and Tobago. The Systemic lupus erythematosus disease activity index (SLEDAI) 2000 score was used for evaluation of activity of the disease. It was found that of all the SLE patients 13 patients $(47 \%)$ were of Afro-Trinidadian descent, 11 patients $(39 \%)$ were of Indo-Trinidadian descent and 4 patients $(14 \%)$ were of mixed descent. The majority of patients were within the 31-35 year age group. Serum IL-17 and IL-23 levels were greater in SLE patients compared to healthy subjects and their levels showed a positive correlation to SLEDAI score. However further experiments are needed to elucidate the role of IL-17 and IL-23 in SLE patients from Trinidad and Tobago.
\end{abstract}

Keywords: Systemic lupus erythematosus; ELISA; Interleukin-17; Interleukin-23; Trinidad and Tobago

\section{Introduction}

Systemic lupus erythematosus (SLE) is an autoimmune disorder that affects many organs including the kidneys, skin, heart, joints, brain and lungs. SLE shows remissions and exacerbations [1]. The cause of SLE is widely unknown, but a genetic susceptibility along with defects in their immune system plays an important role in the immunopathogenesis of this auto-immune disorder. Antigen-presenting cells take up undigested apoptotic cell materials, which are not removed, leading to an increase in the production of antinuclear antibodies. Females are inclined to having Raynaud phenomenon, greater incidences of relapses, arthritis, low blood cell counts and psychiatric symptoms as opposed to males that have more renal disease, seizures, skin problems, serositis and peripheral neuropathy $[2,3]$.

Many studies have been done around the world concerning cytokines and SLE. In one study done by Wong et al. showed that production of IL-17 and IL-23 was uncharacteristically higher in SLE patients compared with non-SLE patients [4]. Similar to this study another one was done by Vincent et al. that showed serum levels of IL-17 were higher in SLE patients than controls, however these interleukin levels had no correlation with the SLEDAI score [5]. The measurements of IL-17 and IL-23 in the blood of patients suffering from SLE in Trinidad and Tobago have never been studied. The aims of this study were: (1) to investigate the serum levels of IL-17 and IL23 in patients with SLE to clarify the role of these cytokines in the immunopathogenesis of this disease and (2) to establish if there is association between the investigated cytokines and the SLEDAI score.

\section{Materials and Methods}

\section{Population, study design and setting}

Twenty eight (28) patients attending the rheumatoid clinic at San Fernando General Hospital were recruited. There was no interventional measure but the study sought to observe and delineate the presence of IL-17 and IL-23 among patients attending the rheumatoid out-patient clinic. Using the Revised American College of Rheumatology (ACR) criteria, diagnosis of SLE was confirmed, and disease activity was assessed by the SLE disease activity index (SLEDAI) 2000 score. A score of $\geq 3$ was considered active disease and a score of $<3$ inactive disease. Patients with active disease $(\mathrm{n}=28)$ and normal non-SLE subjects $(\mathrm{n}=14)$ were recruited.

The characteristics of the study population included all adult females with the diagnosis of active SLE. Female patients were primarily enlisted for the study because the prevalence of the disease is higher in females than in males [3]. Patients over the age of 18, with active SLE disease and of all ethnic groups who give their permission to participate in the project were also included.

\section{Methodology}

All patients with diagnosis of SLE who attend the Rheumatologic clinics at the San Fernando General Hospital were asked to participate in the study after giving their written consent. The basic demographic data of these patients were collected during this period using a standardized questionnaire. Following this, $5 \mathrm{ml}$ of venous blood sample were drawn from their arms. This was taken to Immunology laboratory of the Department of Paraclinical sciences of the University of the West Indies.

Enzyme linked immunosorbent assay (ELISA) for determining the serum concentration of IL-17 or IL-23

ELISA kits were further carried out to determine the presence of IL-17 or IL-23 according to established immunochemical analysis and

*Corresponding author: Angel Justiz-Vaillant, Department of Para-Clinical Sciences, Faculty of Medical Sciences, The University of the West Indies, Trinidad and Tobago, West Indies, Tel: +868-773-5914; E-mail: angel.vaillant@sta.uwi.edu

Received February 12, 2016; Accepted February 27, 2016; Published March 03, 2016

Citation: Nobee A, Justiz-Vaillant A, Akpaka PE, Poon-King P (2016) Levels of Interleukin 17 and 23 in Patients with Systemic Lupus Erythematosus (SLE) in Trinidad and Tobago. Immunochem Immunopathol 2: 115. doi: 10.4172/24699756.1000115

Copyright: ( 2016 Nobee A, et al. This is an open-access article distributed under the terms of the Creative Commons Attribution License, which permits unrestricted use, distribution, and reproduction in any medium, provided the original author and source are credited. 
manufacturer's instructions (IBL corporation, Germany). Ninety-sixwell ELISA plates were already coated with monoclonal anti-human antibodies to either IL-17 or IL-23. Patient samples were added to the plates, incubated and washed 4 times. The wells were incubated with a biotin conjugated anti-human IL-17 or IL-23. The plates were washed again and a peroxidase-labeled streptavidin was added. After a further washing procedure a substrate solution reactive was added. The level of IL-17 or IL-23 present in the sample was proportional to the coloured product developed. The addition of acid stopped the reaction and the absorbance was measured at $450 \mathrm{~nm}$.

\section{Statistical analysis}

Data was stored and analysed using EXCEL and SPSS (version 17) computer software programs. The data was descriptive and reported as comparisons of frequency distributions. A $p$ value of $<0.05$ was considered significant. Lineal regression test was used to determine the concentration of cytokines in the stated population.

\section{Results and Discussion}

Of all the SLE patients 13 (47\%) patients were of Afro-Trinidadian descent, $11(39 \%)$ of patients were of Indo-Trinidadian descent and $4(14 \%)$ of patients were of mixed descent. Of all the SLE patients recruited in this study 1 (4\%) male patient and 27 (96\%) female patients were sampled.

The majority of patients (87\%) experienced symptoms of arthritis, none of patients experienced cardiovascular accidents and the least number of patients experienced urinary problems. Visual disturbances accounted for $33 \%$ of patients, while headaches were experienced for $66 \%$ of them. Vasculitis accounted for $30 \%$ of individuals while renal symptoms such proteinuria and haematuria were seen in $47 \%$ and $27 \%$ of patients respectively. Many experienced symptoms of myositis (77\%) and alopecia (60\%). Pleurisy accounted for $43 \%$ of patients while low complement and leukopenia values were seen in $57 \%$ and $33 \%$ of patients respectively. The $70 \%$ of patients experienced fever.

Both Tables 1 and 2 show that the levels of serum IL-17 and IL-23 were greater in SLE patients when compared to controls $(\mathrm{P}<0.05)$. Table 3 shows that there was no correlation between serum IL-17 or IL-23 in SLE patients $(r=0.276, p>0.05)$. Table 4 shows that serum IL- 17 and IL-23 levels have a positive correlation to SLEDAI score. The Pearson correlation showed that there was no correlation or association between IL-17 and IL-23 suggesting the hypothesis that these cytokines may act in the immunopathogenesis of SLE by independent mechanisms of action rather than jointly and this is supported by the cytokine associations with the SLEDAI score that was statistically significant, suggesting the association of these cytokines with autoimmunity.

In a study by Hegab et al. reported that the IL-23/IL-17 axis could contribute significantly toward the pathogenesis of SLE. IL-23 can promote SLE disease severity, activity, and renal damage by activation of pathogenic Th17 cells. Targeting of IL-23 could be a potential therapeutic approach to treat autoimmune diseases including SLE [6]. Huang et al. studied cytokines in SLE and suggest that upregulation of IL-23 may contribute toward the pathogenesis of SLE [7].

Cheng et al. reported that decreased plasma IL-22 levels correlates with disease activity in SLE patients, but not increased IL-17 and IL23 levels. This discrepancy could be attributed to the different drug regimens used in SLE [8]. In a study done by Mok et al. showed that SLE patients had higher serum IL-17 levels than healthy controls. Elevated serum IL-23 was higher in patients with inflammatory manifestations. The lack of correlation between Th17, Th1, and Th2 cytokines suggested independent regulatory mechanisms for these cytokines [9]. IL-23 has been reported to increase IL-17 secretion in healthy individuals and the results of this research and other findings reported by other authors suggest that the IL-17/IL-23 axis is very much involved in autoimmunity [10]. Furthermore IL-23 plays an important role in the development of pathogenic Th17 cells that synthesizes IL-17 that induces the production of IL- 6 and tumour necrosis factor- $\alpha$, in addition IL-17 causes the attraction of neutrophils to tissues, which are implicated in the SLE pathogenesis $[11,12]$.

Du et al. reported that IL-23 mRNA levels were greater in patients with SLE as well as SLE patients with renal complications, compared with healthy controls. IL-23 mRNA levels showed no difference with active or inactive disease, but in active disease the IL-17/IL-23 ratio was greater. IL-17 and IL-23 mRNA levels were strongly correlated [13].

In conclusion, it was found that serum IL-17 and IL-23 levels were greater in SLE patients compared to controls. Serum IL-17 and IL-23 levels show a positive correlation to SLEDAI score. Further experiments are needed to elucidate the role of IL-17 and IL-23 in SLE patients from Trinidad and Tobago.

\begin{tabular}{|c|c|c|c|c|c|c|c|}
\hline Patient No. & $\begin{array}{c}\text { IL-17 conc. } \\
\text { (pg/ml) }\end{array}$ & $\begin{array}{c}\text { IL-23 conc. } \\
\text { (pg/ml) }\end{array}$ & $\begin{array}{l}\text { SLEDAI } \\
\text { 2K score }\end{array}$ & Patient No. & $\begin{array}{c}\text { IL-17 conc. } \\
\text { (pg/ml) }\end{array}$ & $\begin{array}{c}\text { IL-23 conc. } \\
(\mathrm{pg} / \mathrm{ml})\end{array}$ & $\begin{array}{l}\text { SLEDAI 2K } \\
\text { score }\end{array}$ \\
\hline 1 & 67 & 97.9 & 12 & 15 & 78.8 & 240.1 & 12 \\
\hline 2 & 142 & 92.2 & 11 & 16 & 253.2 & 145.8 & 11 \\
\hline 3 & 292 & 415.8 & 9 & 17 & 92.5 & 143.6 & 14 \\
\hline 4 & 30 & 567.2 & 11 & 18 & 88.2 & 164.4 & 9 \\
\hline 5 & 125 & 310.8 & 12 & 19 & 92.5 & 42.9 & 10 \\
\hline 6 & 64 & 449.4 & 6 & 20 & 29.1 & 131.5 & 5 \\
\hline 7 & 12 & 207.2 & 13 & 21 & 63.1 & 203.6 & 8 \\
\hline 8 & 61 & 430.8 & 4 & 22 & 61.3 & 37.9 & 5 \\
\hline 9 & 0 & 256 & 12 & 23 & 30.8 & 111.5 & 7 \\
\hline 10 & 42 & 491.5 & 11 & 24 & 60.8 & 170.1 & 10 \\
\hline 11 & 0 & 491.5 & 10 & 25 & 56.8 & 185.8 & 13 \\
\hline 12 & 329 & 107.9 & 4 & 26 & 52.2 & 100.1 & 11 \\
\hline 13 & 242 & 60.1 & 7 & 27 & 17.3 & 176.5 & 8 \\
\hline 14 & 197 & 201.5 & 8 & 28 & 80.3 & 107.8 & 10 \\
\hline
\end{tabular}

Table 1: Cytokine concentrations and SLEDAI score among SLE patients. 
Citation: Nobee A, Justiz-Vaillant A, Akpaka PE, Poon-King P (2016) Levels of Interleukin 17 and 23 in Patients with Systemic Lupus Erythematosus (SLE) in Trinidad and Tobago. Immunochem Immunopathol 2: 115. doi: 10.4172/2469-9756.1000115

Page 3 of 3

\begin{tabular}{|c|c|c|c|}
\hline Patient no. & IL-17 conc. (pg/ml) & IL-23 conc. (pg/ml) & SLEDAI 2K score \\
\hline 1 & 0 & 0 & 0 \\
\hline 2 & 0 & 0 & 0 \\
\hline 3 & 20 & 0 & 0 \\
\hline 4 & 0 & 50.1 & 0 \\
\hline 5 & 0 & 0 & 0 \\
\hline 6 & 0 & 91.5 & 0 \\
\hline 7 & 0 & 0 & 0 \\
\hline 8 & 63 & 0 & 0 \\
\hline 9 & 0 & 0 & 0 \\
\hline 10 & 0 & 0 & 0 \\
\hline 11 & 0 & 0 & 0 \\
\hline 12 & 0 & 0 & 0 \\
\hline 13 & 0 & 0 & 0 \\
\hline 14 & 0 & 0 & 0 \\
\hline
\end{tabular}

Table 2: Cytokine concentrations and SLEDAI score among non-SLE patients.

\begin{tabular}{|c|c|}
\hline & Interleukin 17 and Interleukin 23 \\
\hline Pearson correlation $(r)$ & 0.276 \\
\hline$P \quad$ & 0.077 \\
\hline \multicolumn{2}{|c|}{${ }^{*} p$ value shows significance<0.05 level } \\
\hline
\end{tabular}

Table 3: Association between Interleukin 17 and interleukin 23.

\begin{tabular}{|c|c|c|c|c|}
\hline & \multicolumn{2}{|c|}{ Interleukin 17 } & \multicolumn{2}{c|}{ Interleukin 23 } \\
\hline & $\mathrm{R}$ & $\mathrm{P}$ & $\mathrm{R}$ & $\mathrm{p}$ \\
\hline SLEDAI & 0.371 & 0.016 & 0.512 & 0.001 \\
\hline \multicolumn{3}{|c|}{${ }^{*} \mathrm{p}$ value shows significance<0.05 level } \\
\hline
\end{tabular}

Table 4: Association between cytokines and SLEDAI score.

\section{References}

1. Rahman A, Isenberg DA (2008) Systemic lupus erythematosus. N Engl J Med 358: 929-939.

2. Lisnevskaia L, Murphy G, Isenberg D (2014) Systemic lupus erythematosus Lancet 384: 1878-1888.

3. Yacoub Wasef SZ (2004) Gender differences in systemic lupus erythematosus Gend Med 1: 12-17.

4. Wong CK, Lit LC, Tam LS, Li EK, Wong PT, et al. (2008) Hyperproduction of IL-23 and IL-17 in patients with systemic lupus erythematosus: implications for Th17-mediated inflammation in auto-immunity. Clin Immunol 127: 385-393.

5. Vincent FB, Northcott M, Hoi A, Mackay F, Morand EF (2013) Clinica associations of serum interleukin-17 in systemic lupus erythematosus. Arthritis Res Ther 15: R97.

6. Hegab S, Mahmoud G, Mohamed S, Abou A, Mohamed E, et al. (2014) Role of interleukin-23 in the immunopathogenesis of systemic lupus erythematosus. The Egyptian Journal of Dermatology and Venereology 34: 120-125.

7. Huang X, Hua J, Shen N, Chen S (2007) Dysregulated expression of interleukin-23 and interleukin-12 subunits in systemic lupus erythematosus patients. Mod Rheumatol 17: 220-223.

8. Cheng F, Guo Z, Xu H, Yan D, Li Q (2009) Decreased plasma IL22 levels, but not increased IL17 and IL23 levels, correlate with disease activity in patients with systemic lupus erythematosus. Ann Rheum Dis 68: 604-606.

9. Mok MY, Wu HJ, Lo Y, Lau CS (2010) The relation of interleukin 17 (IL17) and IL-23 to Th1/Th2 cytokines and disease activity in systemic lupus erythematosus. J Rheumatol 37: 2046-2052.

10. Hoeve MA, Savage ND, de Boer T, Langenberg DM, de Waal Malefyt R, et al (2006) Divergent effects of IL-12 and IL-23 on the production of IL-17 by human T cells. Eur J Immunol 36: 661-670.

11. Cornelissen F, van Hamburg JP, Lubberts E (2009) The IL-12/IL-23 axis and its role in Th17 cell development, pathology and plasticity in arthritis. Curr Opin Investig Drugs 10: 452-462.

12. Bettelli $E$, Oukka M, Kuchroo VK (2007) $T(H)-17$ cells in the circle of immunity and autoimmunity. Nat Immunol 8: 345-350.

13. Du J, Li Z, Shi J, Bi L (2014) Associations between serum interleukin-23 levels and clinical characteristics in patients with systemic lupus erythematosus. J Int Med Res 42: 1123-1130. 